\title{
Comparación de dos Softwares en Histología Humana, como Utilización Complementaria a la Enseñanza Tradicional
}

\author{
Comparison of Two Human Histology Software as Complementary Use in Traditional Education
}

\author{
Carolina Schencke \& Alejandra Hidalgo
}

SCHENCKE, C. \& HIDALGO, A. Comparación de dos softwares en Histología Humana, como utilización complementaria a la enseñanza tradicional. Int. J. Morphol., 29(4):1389-1393, 2011.

RESUMEN: Actualmente, la formación de profesionales en la Educación Superior desafía a los docentes a incorporar en sus prácticas de enseñanza no solamente el dominio de contenido disciplinario puro, además, les exige competencias asociadas a la apropiación de conocimientos didácticos para guiar el saber disciplinario. El siguiente estudio pretende aportar con nuevas metodologías de aprendizaje en forma de softwares didácticos para los estudiantes que cursan la asignatura de Histología, de la carrera de Enfermería, Facultad de Ciencias de Salud de la Universidad Autónoma de Chile. Estos módulos interactivos se crearon como apoyo a las clases tradicionales de pasos prácticos con observación al microscopio. Para desarrollar el primer módulo, un total de 200 imágenes fueron digitalizadas a formato JPG. A partir de estas imágenes se realizaron presentaciones con animación personalizada, la que fue llevada al programa Adobe Flash Player creando así al primer software titulado "Módulo Histológico". Al módulo inicial incorporamos información funcional de las preparaciones histológicas, además de mapas conceptuales. Esta innovación resultó especialmente atractiva y decidimos modificar el primer software, creando el segundo programa titulado "Modulo Histofuncional". Para comparar la percepción de los estudiantes sobre ambos módulos se realizó una encuesta de opinión voluntaria y anónima, previamente validada, que contenía preguntas sobre factores relacionados con: contenido, motivación, utilidad, calidad y cantidad. Las respuestas fueron evaluadas a través de una escala de Likert. Los resultados reflejaron objetivamente la recepción favorable que ha tenido el Modulo Histofuncional por parte de nuestros estudiantes. Esto, probablemente, debido a que este software logra integrar el conocimiento de las estructuras con su función, entregando significancia, facilitando el aprendizaje y motivando a los alumnos en el estudio de la Histología.

PALABRAS CLAVE: Histología; Docencia; Computación; Enseñanza.

\section{INTRODUCCIÓN}

Actualmente, la formación de profesionales en la Educación Superior desafía a los docentes a incorporar en sus prácticas de enseñanza no solamente el dominio de contenido disciplinario puro, sino que, además, les exige competencias asociadas a la apropiación de conocimientos didácticos para vehiculizar el saber disciplinario hacia el saber pedagógico, con el objeto de cumplir con el acto educativo (Pellón et al., 2009). Estos mismos, hicieron posible constatar que la sabiduría didáctica práctica es una fuente de conocimiento relevante en el aprendizaje. De esta manera es necesario valorizarla como el principal conocimiento base para la formación en carreras de la Salud (Pellón et al., 2010).

Estudios realizados sobre el aprendizaje significativo en las Ciencias Morfológicas (Dionisio de Cavalier \&
Chalub, 2009) demuestran que el valor de este aprendizaje radica en los conocimientos en base al sentido que el estudiante pueda dar a aquello que "nombra, reconoce o describe". La Histología es una disciplina que pertenece a las Ciencias Morfológicas donde la estructura de células, tejidos y órganos del cuerpo son examinados microscópicamente. La metodología utilizada tradicionalmente en la enseñanza práctica de la Histología se basa en el uso de microscopios y preparaciones histológicas. Sin embargo, esta metodología ha resultado para muchas generaciones de alumnos árida y poco motivadora (Rojas et al., 1999). Esto se debe, probablemente, a que los microscopios ópticos no permiten la observación por varias personas al mismo tiempo, lo que dificulta la discusión interactiva. En el proceso del aprendizaje morfológico, deben usarse todos los métodos disponibles, no sólo como medio para determinar la aprobación de 
la asignatura, sino, como recurso para motivar y facilitar el proceso de aprendizaje (García-Hernández, 2006). Generar metodologías de enseñanza apropiadas para la morfología a sido tema de gran interés y han generado discusión en los últimos años, (Galván et al., 2000; Guiraldes et al., 2001; Cabalin et al., 2002; Inzunza et al., 2003; Suazo, 2007).

Hoy en día, los avances en informática permiten reproducir imágenes proyectadas al microscopio y llevarlas al monitor de los computadores donde los alumnos la visualizan en forma cómoda y objetiva, facilitando el adiestramiento en el reconocimiento de las estructuras. Ávila et al., (2010), menciona que la informática e Internet contribuyen a asociar por un lado el almacenamiento, gestión, y suministro de grandes volúmenes de información y por el otro, la posibilidad de usar entornos de aprendizajes interactivos y autorregulados. Galván et al., (1999) nos indica que la utilización de recursos computacionales ha incidido de manera benéfica, ya que los alumnos reciben con agrado la información visual. En general, la computación ha beneficiado la educación en carreras de la Salud, ya que ha logrado generar un aprendizaje interdisciplinario, realizar lecturas más interesantes por presentaciones en multimedia, reducir el tiempo que necesitan los estudiantes en buscar la información y generar interacción con el estudiante, (Dantas \& Martins, 2005). En Histología los programas computacionales no pueden reemplazar las actividades de observación al microscopio, sin embargo, facilitan el aprendizaje. El uso de imágenes digitalizadas permite focalizar y facilitar el aprendizaje del alumno, siendo este un excelente medio de enseñanza previo a la observación de un tejido complejo a través del microscopio (Rojas et al.).

Los programas computacionales tienen una buena aceptación entre alumnos, sirven de instancia de repaso o de preparación para el paso práctico, sin embargo, estos programas no reemplazan las clases teóricas ni la lectura programada de los textos guías (Inzunza \& Bravo, 1999; Inzunza et al., 2003). Quienes promueven la integración de las nuevas tecnologías de información y comunicación para el aprendizaje de las ciencias, si pueden decir que los diversos medios informáticos y electrónicos, desarrollados y utilizados adecuadamente, favorecen un mayor despliegue de estrategias cognitivas en el tratamiento de la información, (Waldegg, 2002).

Cuando la información se obtiene de la visualización de una imagen esta se interpreta en forma mas fidedigna a la que se registra a partir de un texto. "Una imagen vale más que 1000 palabras", es decir, es un recurso con elevado poder pedagógico, a través de la cual se puede orientar al alumno a realizar acciones para la adquisición de conocimientos.
Sin embargo las imágenes crudas, no tratadas dicen poco, pueden desorientar al alumno, y a veces al docente, (Galván et al.). Analizar este tipo de imágenes, cuyo aporte es calificado como elemental por alumnos de medicina es como mirar un plano de calles de una ciudad donde no hay hitos claros de referencia (Guiraldes et al.; Carranza \& Celaya, 2003). Es por este motivo la importancia de aportar en la enseñanza morfológica con elementos metodológicos suficientemente interactivos.

Con la influencia de la informática y como consecuencia de la era de Internet, las comunicaciones están cambiando con una rapidez mucho mayor que la imaginada, estimulando a las instituciones docentes a hacer uso de dichas herramientas. Gutiérrez, 1987, describe que los computadores y la multimedia son herramientas que permiten el aprendizaje concebido como una construcción que realiza el alumno en su interacción con el medio, o al relacionar nuevas ideas con las que el alumno posee en forma previa. En este sentido, aprender es un proceso que se asimila al de pensar en la medida que implica partir de lo que el sujeto ya sabe (conocimientos previos) y aplicar estrategias específicas sobre una situación que se plantea como un problema a interpretar e intervenir.

En nuestra Facultad, sede Temuco, hemos creado módulos interactivos en forma de softwares didácticos, como apoyo multimedial a las clases tradicionales de pasos prácticos con observación al microscopio para la asignatura de Histología, carrera de Enfermería. La construcción del $1^{\circ}$ objeto de aprendizaje titulado "Modulo Histológico" se realizó en conjunto con alumnos de la carrera de Enfermería de $1^{\circ}$ año de nuestra Facultad, sede Temuco, los que se inscribieron voluntariamente. Asistieron a la sala de computación, donde se realizaron discusiones sobre las ventajas y desventajas del módulo creado en base a imágenes en formato power point. Los estudiantes solicitaron mayor tiempo para trabajar con el módulo Histológico, e incorporar más información en relación a las funciones de cada estructura, ya sea en forma de mapas conceptuales o de esquemas representativos. Esta innovación nos resultó especialmente atractiva y decidimos modificar nuestro primer software, generándose el "Módulo Histofuncionl". El segundo programa se encuentra actualmente en el servidor SAGAF de nuestra Universidad, a libre disposición de nuestros alumnos.

El presente estudio pretende lograr un mejoramiento de los aprendizajes histológicos de los estudiantes de la Facultad de Ciencias de Salud, de la Universidad Autónoma de Chile, durante las actividades prácticas, al incorporar nuevas metodologías de aprendizaje en forma de programas interactivos. 


\section{MATERIAL Y MÉTODO}

En la Facultad de Ciencias de la Salud, Sede Temuco, de la Universidad Autónoma de Chile, se construyó el primer modulo de aprendizaje histológico en base a fotografías obtenidas de las muestras usadas para docencia y muestras obtenidas en actividades de investigación. Para la obtención de fotografías de preparados histológicos se utilizó un microscopio Carl Zeiss, Axiolab, con cámara Mc80 DX. Se fotografiaron los mismos preparados histológicos que el estudiante debe observar durante los pasos prácticos de microscopia. Un total de 200 imágenes fueron digitalizadas a formato JPG. A partir de estas imágenes se realizaron presentaciones power point con animación personalizada, la que fue finalmente llevada al programa Adobe Flash Player.

Ambos instrumentos didácticos brindan al usuario un conjunto de imágenes a color sobre la histología de los tejidos básicos, conformados por tejido epitelial, conjuntivo, muscular y nervioso; y por los sistemas circulatorio, urinario, respiratorio, endocrino, digestivo y reproductor. El Módulo Histológico contiene fotos de las preparaciones en distintos aumentos y cuyas estructuras se encuentran identificadas. El Módulo Histofuncional contiene fotos de las preparaciones en distintos aumentos, con estructuras identificadas, en la que se describe la función de cada estructura, órgano o sistema. Se incorporó a este modulo una gran variedad de ilustraciones originales que permitieron evidenciar y visualizar los conceptos.

La actividad de evaluación de los módulos de aprendizaje se realizó con estudiantes de la carrera de Enfermería, que cursaban la asignatura de Histología. Este trabajo involucró las actividades de apoyo a la docencia, SAAC (Sistema de Apoyo Académico Complementario) realizándose durante el trabajo de ayudantías. El número de alumnos que evaluaron directamente los módulos fue de 100. Los alumnos tuvieron acceso a ambos módulos a través del servidor SAGAF (Sistema de Apoyo Académico de Gestion y Financiero) de nuestra casa de estudios y en los laboratorios de computación de esta Facultad.
Para el acceso al módulo en los laboratorios de computación de la Facultad de Ciencias de Salud, se grabaron ambos módulos en un computador servidor, al cual se conectaron 30 máquinas remotas de marca Samsung, (Pentium de 1.67 GHZ, disco duro de $40 \mathrm{Mb}$ de RAM, monitor de 13 pulgadas), teniendo acceso a un archivo compartido a través de un número IP por medio de una red local.

Para evaluar los módulos, los estudiantes realizaron una encuesta de opinión voluntaria y anónima, previamente validada, que contenía preguntas sobre factores relacionados con: contenido, motivación, utilidad, calidad y cantidad. Las respuestas fueron evaluadas a través de una escala de Likert con cinco posibilidades: totalmente en desacuerdo, en desacuerdo, indiferente, de acuerdo y totalmente de acuerdo, con una escala del 1 al 5, respectivamente.

\section{RESULTADOS}

La experiencia pedagógica con los módulos interactivos evidenció una inmediata satisfacción con respecto al Módulo Histofuncional. En relación a este programa, sobre el $70 \%$ de los alumnos se encontró totalmen-

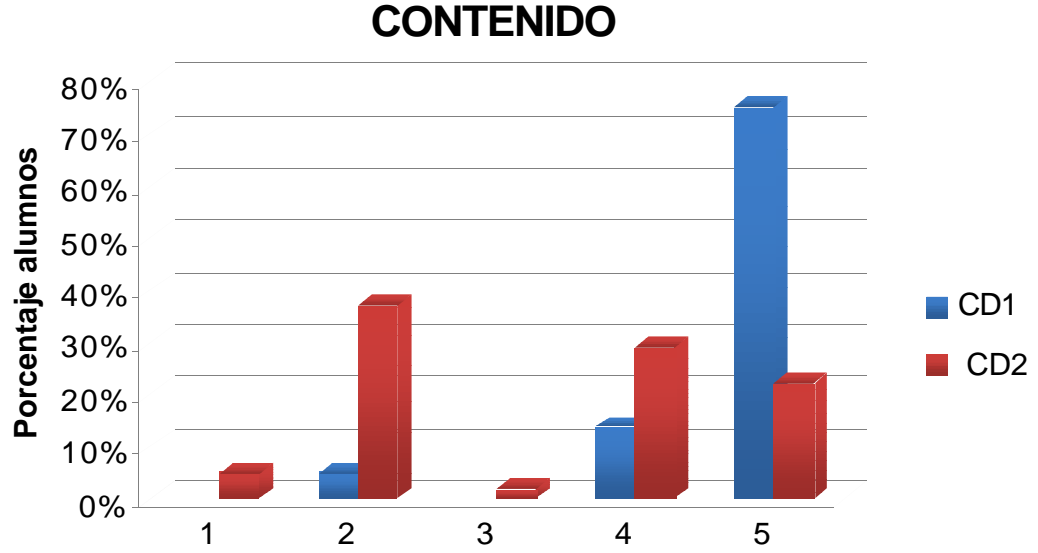

Fig. 1. Opinión de los alumnos de la carrera de Enfermería, sede Temuco, Universidad Autónoma de Chile, en relación a los contenidos de los programas computacionales: Módulo Histofuncional (azul), Modulo Histológico (rojo)

\section{MOTIVACIÓN}

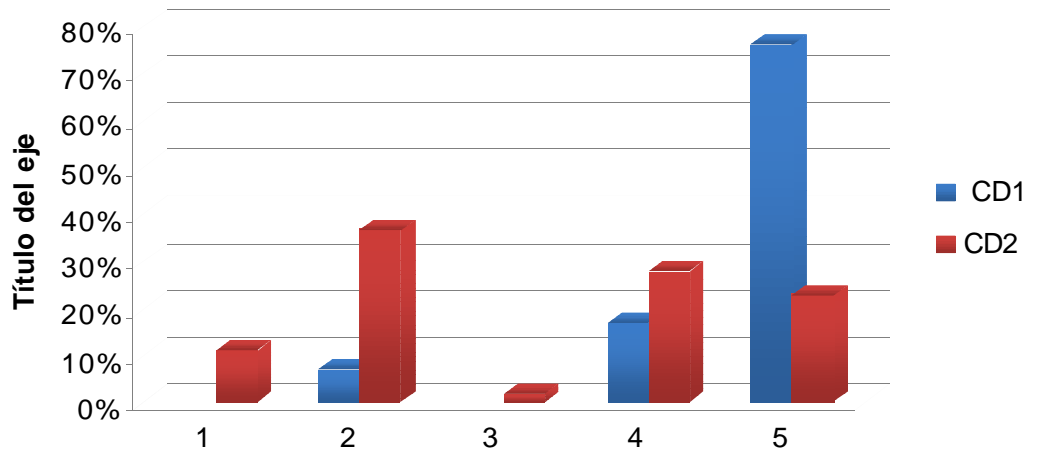

Fig. 2. Opinión de los alumnos de la carrera de Enfermería, sede Temuco, Universidad Autónoma de Chile, en relación a la motivación por estudiar con el módulo Histofuncional (azul) e Histológico (rojo) como recurso de aprendizaje. 


\section{CALIDAD}

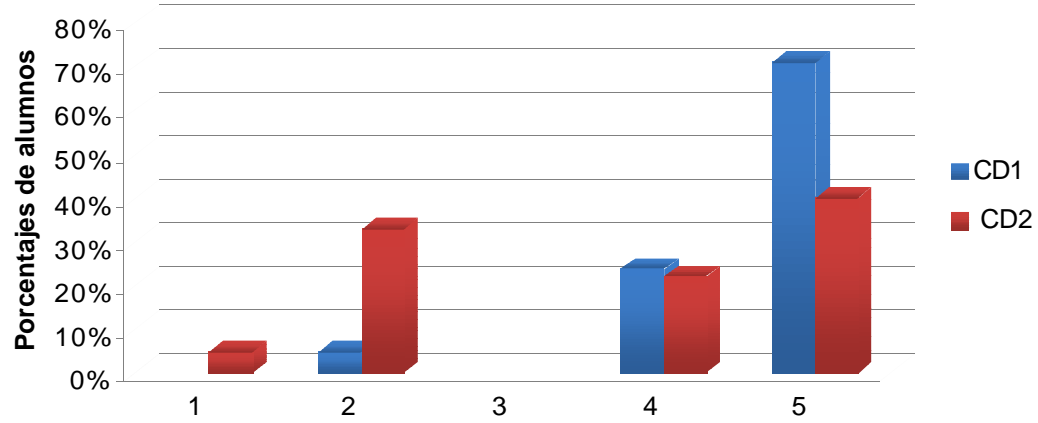

Fig. 3. Opinión de los alumnos de la carrera de Enfermería, sede Temuco, Universidad Autónoma de Chile, en relación a la calidad de la información y del material presente en el Módulo Histofuncional (azul) e Histológico (rojo).

\section{CANTIDAD}

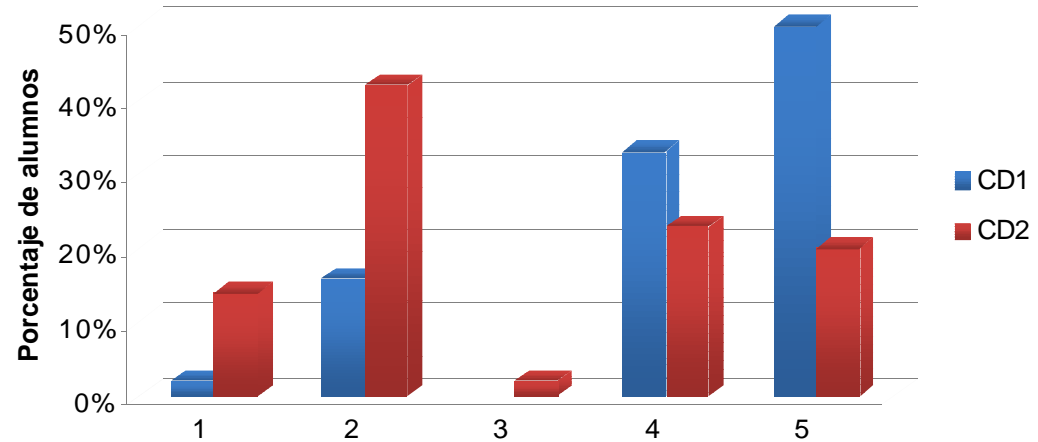

Fig. 4. Opinión de los alumnos de la carrera de Enfermería, sede Temuco, Universidad Autónoma de Chile, en relación a la cantidad de información

\section{UTILIDAD}

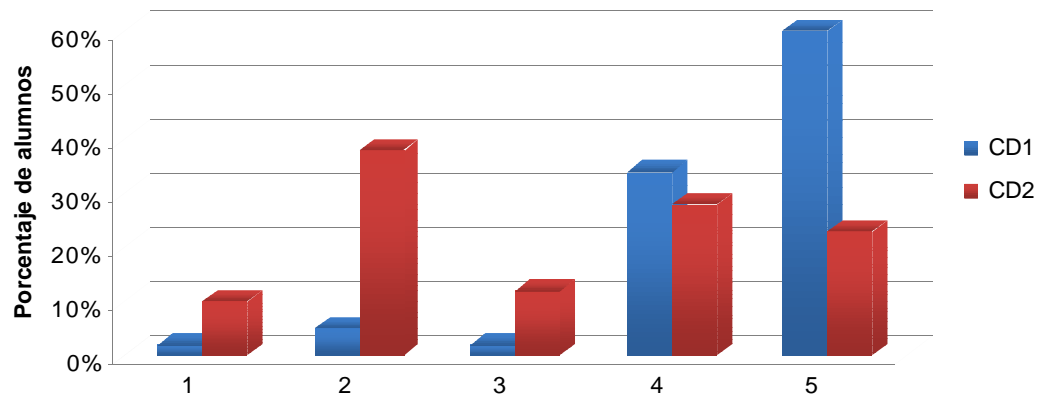

te de acuerdo en relación al contenido y buena calidad de la información. La motivación por estudiar con este programa fue mayor al $80 \%$. Sin embargo, cerca del $40 \%$ de los alumnos se encontró en desacuerdo en relación a la motivación que genera el programa Módulo Histológico y a su contenido, comentando que este programa no logra mayor interacción. (Figs. 1, 2 y 3). Los alumnos solicitaron mayor cantidad de información para ambos programas interactivos. El $45 \%$ de los alumnos se encontró en desacuerdo en relación a la cantidad de información entregada por el Módulo Histológico y solo el $50 \%$ de los alumnos estuvo totalmente de acuerdo con la cantidad de información entregada por el Módulo Histofuncional. En este último solicitaron mayor cantidad de esquemas y mapas conceptuales relacionados con las fotos histológicas.

La utilidad del recurso Módulo Histofuncional es altamente valorada por los estudiantes, quienes comentan que lograron corroborar sus conocimientos y generar mayor interacción con este programa.

\section{DISCUSIÓN}

El aprendizaje y conocimiento de las Ciencias Morfológicas requiere no sólo de la asimilación de conceptos y del dominio de habilidades, sino fundamentalmente, de la capacidad de otorgar sentido, establecer relaciones entre conocimientos y desarrollar prácticas orientadas por la comprensión significativa entre los conocimientos teóricos y las evidencias prácticas de la morfología celular.

Para que cada concepto pueda ser aprendido significativamente, el estudiante debe ser capaz de entender o comprender el significado. En términos de David Ausubel, algo tiene significado cuando se logra entender y darle sentido. Un estudiante comprende el significado de los términos teóricos cuando puede definirlos y explicarlos. En relación a lo anterior, el Espacio Europeo de Educación Superior (De Miguel) reconoce tácitamente los principios tradicionalistas de enseñanza, señalando la necesidad de un cambio de paradigma que apunta a que sea el propio alumno el que asuma la organización y desarrollo de su trabajo académico. 
Durante las sesiones de observación de ambos programas interactivos, se logró interactuar en relación a la materia, logrando, incluso, concluir opiniones unánimes entre la alumna ayudante y los estudiantes. Se abrieron instancias para que el alumno se sintiera libre de preguntar. El trabajo con los módulos facilitó el proceso de comprensión, ya que todos observaron la imagen al mismo tiempo. Se generó una interesante interacción entre alumno-ayudantedocente, a través de la formación de preguntas que ellos mismos respondieron gracias a la discusión generada.

La mayoría de los softwares que desarrollan los contenidos de las disciplinas de anatomía e histología, lo hacen con un criterio no integrado, donde los conceptos se abordan en forma separada, independiente de la funcionalidad de la estructura, (Carranza \& Celaya; Guiraldes et al.). Basándonos en estos autores, y en la opinión de los estudiantes, se generó la implementación de un nuevo recurso de estudio, que se llamó Módulo Histofuncional.

El marco conceptual utilizado para la concepción del Módulo Histofuncional se basó en emplear estrategias más dinámicas y participativas, cuya ventaja fue combinar la información visual y textual en un recurso mediador. El estudiante se muestra más motivado por trabajar con este segundo módulo, lo que se manifiesta al formular preguntas y respuestas con mayor grado de profundización. La decisión de emplear imágenes combinadas con texto, mapas conceptuales, $\mathrm{y}$ esquemas se ofreció como una estrategia para mejorar la com- prensión, y se basó en las ventajas propias que ofrece el sistema interactivo. De esta forma, se establecen enlaces asociativos entre el texto y las imágenes, según lo sugieren los diseñadores de visualización (Carranza \& Celaya).

La diferencia entre el aprendizaje de representación y el de conceptos, es que en éste último, se incorporan los atributos de criterio. La estructura cognoscitiva que el alumno aprende en el Módulo Histofuncional se enriquece, ya que, al aprender significativamente un concepto, este se integra activamente y permite una representación simplificada y generalizada de la realidad (Ausubel 1978; Gutiérrez, 1987; Moreira \& Greca 2003; Galagovsky 2004)

En general, los resultados reflejan de un modo bastante objetivo la recepción favorable que ha tenido el Modulo Histofuncional por parte de los estudiantes. Los resultados obtenidos señalan que el estudio integrado de los temas podría facilitar el aprendizaje significativo, así como lo describen los autores Galván et al.; Guiraldes et al.; Cabalin et al.; Inzunza et al. y Suazo.

En este estudio hemos adquirido experiencia en lo que refiere a construir, implementar, y evaluar un recurso didáctico. Este trabajo, además, nos ha proporcionado información necesaria para incorporar mejoras en el proceso de autoaprendizaje de nuestros estudiantes. En este sentido, hemos motivado a los alumnos a convertirse en constructores-editores de materiales de enseñanza.

SCHENCKE, C. \& HIDALGO, A. Comparison of two Human Histology Software as complementary use in traditional education.. Int. J. Morphol., 29(4):1388-1393, 2011.

SUMMARY: At present, the training of professionals in Higher Education challenges docents to incorporate in their teaching practice, not only expertise of pure disciplinary content, it also calls for associated aptitudes of didactic knowledge to guide the disciplinary knowledge. This study intends to contribute new teaching methodologies in the form of didactic software for Histology students in the Nursing Degree program, Faculty of Health Sciences of the Universidad Autónoma De Chile. These interactive modules were created as support for traditional classes and practical steps with microscopic observation. For the development of the first module, a total of 200 images were digitalized in JPG format. From those images presentations with personal animation were realized, which were then converted to Adobe Flash Player thus creating the first software entitled "Histological Module". We incorporated functional information to the initial module of histological preparation in addition to concept maps. This innovation was particularly attractive and we decided to modify the initial software, thereby creating the second program entitled "Histo-functional Module". To compare student perception on both modules a previously validated, voluntary and anonymous opinion survey was carried out that included questions on factors related to: content, motivation, utility, quality and quantity. Responses were evaluated by Likert scale. Results objectively reflected the favorable acceptance that the histo-functional Module has had on the part of our students. This is probably the result of this software integrating knowledge of structures and their function, affording it meaning and facilitating learning as well as motivating students in the study of histology.

KEY WORDS: Histology; Docency; Computer technology; Teaching.

\section{REFERENCIAS BIBLIOGRÁFICAS.}

Ausubel, D. Psicología educativa. Un punto de vista cognocitivo. Trillas, México, 1978.
Ávila., R.; Alonso, L.; Samar, M.; Buzetti, L.; Juri, G \& Juri, H. Ampliaciones de una mediateca digital de mues- 
tras de laboratorio histopatológico. Int. J. Morphol., 28(3):875-8, 2011.

Carranza, M. \& Celaya, G. Una estrategia para favorecer la comprensión y el aprendizaje en las ciencias morfológicas: presentaciones en powerpoint. Relieve., 9(2):139-59, 2003.

Cabalin, D; Collipal, E. \& Doussoulin, A. Evaluación de módulos de autoaprendizaje en anatomía humana. Rev. Chil. Anat., 20(2):211-4, 2002.

Dantas, E. \& Martins, J. Students opinion on the use of computer rooms for teaching anatomy. Int. J. Morphol., 23(3):267-70, 2005.

Dionisio de Cavalier, M. \& Chalub, D. El aprendizaje significativo de las ciencias morfológicas en medicina: experiencia y aportes para su enseñanza en clínica dermatológica. Int. J. Morphol., 27(2):565-9, 2009.

Collipal Larré, E. Conceptualización a través de redes semánticas naturales de los módulos de autoaprendizaje en anatomía humana. Rev. Chil. Anat., 20(1):63-7, 2002.

Galagovsky, L. Del aprendizaje significativo al aprendizaje sustentable. Parte 1: El modelo Teórico. Enseñanza de las ciencias, 22(2):229-40, 2004.

Galván, S.M.; Gimeno, M.; Nuviala, J.; Gil, J.; Andreotti, C.; Sbodio, O \& Pastor, R. Potencialidades y limitaciones del uso de recursos multimediales en la enseñanza de anatomía veterinaria. Rev. Chil. Anat., 18(1):75-83, 2000.

Galván, S.M.; Visciglio, S.; Andreotti, C. \& Sbodio, O. Efectos del uso de tecnologías de imagen en el aprendizaje de materiales fácticos en los estudiantes de anatomía veterinaria. Rev. Chil. Anat., 17(1):11-20, 1999.

García-Hernández, F. Evaluación práctica de la anatomía basada en la teoría de las inteligencias múltiples de Gardner, carrera de odontología de la Universidad de Antofagasta, Chile. Int. J. Morphol., 24(1):83-8, 2006.

Guiraldes, H., Oddó, H; Mena, B.; Velasco, N. \& Paulos, J. Enseñanza de la anatomía humana: experiencias y desafíos en una escuela de medicina. Rev. Chil. Anat., 19(2):205-12, 2001.

Gutierrez, R. Psicología y aprendizaje de las ciencias. El Modelo de Ausubel. Enseñanza de las ciencias, 5(2):11828, 1987.
Inzunza, O.; Acuña, E.; \& Bravo, H. Evaluación práctica de anatomía. Rendimiento de los alumnos de primer año de medicina ante distintas formas de preguntar. Int. $J$. Morphol., 21(2):131-6, 2003.

Inzunza, O. \& Bravo, H. Impacto de dos programas computacionales de anatomía humana en el rendimiento del conocimiento practico de los alumnos. Rev. Chil. Anat., 17(2):205-9. 1999.

Moreira, A \& Greca, I. Cambio conceptual: análisis crítico y propuestas a la luz de la teoría del aprendizaje significativo. Ciencia y Educación, 9(2):301-15, 2003.

Pellón, A; Mansilla, J. \& San Martin D. Desafíos para la transposición didáctica y conocimiento didáctico del contenido en docentes de anatomía: obstáculos y proyecciones. Int. J. Morphol., 27(3):743-50, 2009.

Pellón, A; Mansilla, J. \& San Martin D. Importancia de a sabiduría didáctica práctica como fuente de conocimiento base para la enseñanza de la anatomía. Int. J. Morphol., 28(1):219-26, 2010.

Rojas, M.; Montiel, E.; Montiel, J.; Ondarza, A.; Rodríguez, H. Estudio comparativo entre métodos de enseñanza tradicional y computacional en histología humana. Rev. Chil. Anat., 17(1):81-5, 1999.

Suazo, G .I. Estilos de aprendizaje y su correlación con el rendimiento académico en anatomía humana normal. Int. J. Morphol., 25(2):367-73, 2007.

Waldegg, G. El uso de las nuevas tecnologías para la enseñanza y el aprendizaje de las ciencias. Relieve, 4(1):96116, 2002.

Dirección para correspondencia: M. Sc. Carolina Schencke F. Facultad de Ciencias de la Salud Universidad Autónoma de Chile Temuco - CHILE

Email: carolinaschencke@gmail.com

Recibido: 20-07-2011

Aceptado:21-09-2011 